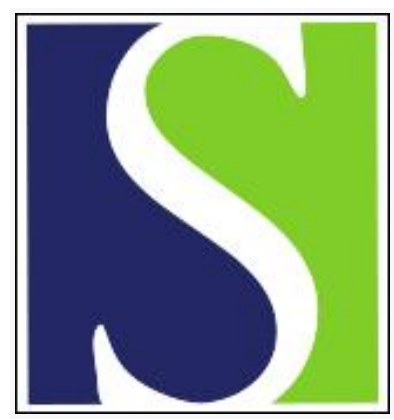

Scand J Work Environ Health 2009;35(1):74-79

https://doi.org/10.5271/sjweh.1303

Issue date: 00 Jan 2009

Shift work, chronodisruption and cancer?-the IARC 2007 challenge for research and prevention and 10 theses from the Cologne Colloquium 2008

by Erren TC, Morfeld P, Stork J, Knauth P, von Mülmann MJA, Breitstadt $\mathrm{R}$, Müller U, Emmerich M, Piekarski C

Affiliation: Institute and Policlinic for Occupational and Social Medicine, School of Medicine and Dentistry, University of Cologne, Kerpener Str. 62, 50937 Köln, Germany. tim.erren@uni-koeln.de

Refers to the following texts of the Journal: 2007;33(5):321-323 2007;33(5):336-343

The following articles refer to this text: $2011 ; 37(1): 54-61$;

2010;36(6):515-516; 2014;40(6):621-630

Key terms: breast cancer; cancer; chronodisruption; circadian disruption; Cologne Colloquium 2008; IARC; International Agency for Research on Cancer; prevention; prostate cancer; prudent avoidance; research; shift work

This article in PubMed: www.ncbi.nlm.nih.gov/pubmed/19277435 


\title{
Shift work, chronodisruption and cancer?-the IARC 2007 challenge for research and prevention and 10 theses from the Cologne Colloquium 2008
}

\author{
by Thomas C Erren, MD, ${ }^{1}$ Peter Morfeld, PhD, ${ }^{2}$ Joachim Stork, MD, ${ }^{3}$ Peter Knauth, PhD, ${ }^{4}$ Mat- \\ thias JA von Mülmann, MD, ${ }^{5}$ Rolf Breitstadt, MD, ${ }^{6}$ Uta Müller, MD, Michael Emmerich, MD, ${ }^{8}$ \\ Claus Piekarski, $M D^{1}$
}

\begin{abstract}
Erren TC, Morfeld P, Stork J, Knauth P, von Mülmann MJA, Breitstadt R, Müller U, Emmerich M, Piekarski C. Shift work, chronodisruption and cancer?-the IARC 2007 challenge for research and prevention and 10 theses from the Cologne Colloquium 2008. Scand J Work Environ Health. 2009;35(1):74-79.

In October of 2007, an IARC panel of 24 scientists systematically evaluated epidemiologic, experimental, and mechanistic data and concluded that shift work that involves circadian or chronodisruption is probably carcinogenic in humans. In view of the possible scope of the problem - shift work is widespread and unavoidable on one hand and breast cancer and prostate cancer, which may be causally associated with chronodisruption, are epidemic worldwide on the other-German representatives of science and occupational medicine discussed the experimental and epidemiologic background and possible implications of the challenge identified by the International Agency for Research on Cancer (IARC) at a colloquium in Cologne in September 2008. This overview summarizes the key ideas presented at the Cologne Colloquium and offers 10 theses concerning the need for targeted studies and the necessity to develop possible means of prevention.
\end{abstract}

Key terms breast cancer; circadian disruption; International Agency for Research on Cancer (IARC); prostate cancer; prudent avoidance.

Following a diligent review of abundant experimental and limited epidemiologic evidence, a panel of 24 scientists from 10 countries convened by the International Agency for Research on Cancer (IARC) recently classified shift work involving circadian disruption as probably carcinogenic to humans (group 2A) (1). With some 15-20\% of the workers in North America and Europe engaged in shift work, predominantly in health care, industry, transportation, communications, and hospitality work, this overall evaluation can have far-reaching consequences.

Intriguingly, the IARC experts identified several biologically plausible lines of mechanistic causation or determination, including "exposure to light at night", a feature common in night work, "disturbs the circadian system with alterations of sleep-activity patterns, suppression of melatonin production and deregulation of circadian genes involved in cancer-related pathways [p 1065]" (1). Straif and his colleagues concluded in Lancet Oncology that "among the many patterns of shiftwork, those including nightwork are the most disruptive for the circadian clock [p 1065]" (1).

Now, biological 24-hour rhythms allow organisms, including humans, to tune physiology to daily cycles of sunlight and darkness, of day and night. That proper circadian regulation is essential for the well-being of organisms is beyond doubt (2). Importantly, central timing and an appropriate coordination of circadian rhythms is

Institute and Policlinic for Occupational and Social Medicine, School of Medicine and Dentistry, University of Cologne, Köln, Germany.

2 Institute for Occupational Epidemiology and Risk Assessment of Evonik Industries AG, Essen, Germany.

3 Gesundheitswesen, AUDI AG, Ingolstadt, Germany. Institut für Industriebetriebslehre und Industrielle Produktion (IIP), Universität Karlsruhe (TH), Karlsruhe, Germany. Medizinischer, Dienst, Deutsche Lufthansa AG, Frankfurt, Germany. C-ES-OH Umwelt, Sicherheit, Gesundheit Evonik Industries AG, Frankfurt, Germany. C-ES-OH Umwelt, Sicherheit, Gesundheit Evonik Industries AG, Essen, Germany. Arbeitsmedizinisches Zentrum Hirschbach, RAG Deutsche Steinkohle, Sulzbach-Saar, Germany.

Correspondence to: Dr TC Erren, Institute and Policlinic for Occupational and Social Medicine, School of Medicine and Dentistry, University of Cologne, Kerpener Str. 62, 50937 Köln, Germany. [tim.erren@uni-koeln.de] 
achieved by a circadian master clock in the brain, which collectively sets or governs - similar to Greenwich mean time, which provides the reference time, independent of the geographic location, for billions of other clocks in the world-a plethora of peripheral clocks. In doing so in response to ambient light, the circadian master clock coordinates tissue- and organ-specific 24-hour rhythms that would otherwise act independently of one another (3). The light-dependent master clock or master circadian pacemaker was identified in the early 1970s, namely, in the suprachiasmatic nuclei of the anterior hypothalamus (4), and has been qualified thereafter as a tumor suppressor (5). Failures to coordinate peripheral rhythms via the suprachiasmatic nuclei can lead to circadian or chronodisruption, a relevant disturbance of the circadian organization of physiology, endocrinology, metabolism, and behavior, which links light exposure, shift work, biological 24-hour rhythms, and the development of internal cancers in a biologically plausible way. [An overview of the experimental work can be found in a paper by Erren et al (6).]

Clearly, the experimental and mechanistic evidence for biologically plausible causal links is compelling and has been the driving force for the IARC classification. Equally clearly, the epidemiologic evidence to date is too limited to contribute much to causal considerations.

Remarkably, today's compelling experimental evidence on which IARC primarily based their group 2A classification provides an unusual situation for epidemiologic researchers. Indeed, in many-if not most-instances, epidemiologists have to embark on so-called black-box epidemiology (7) (ie, we may suspect that there is a causal mechanism but its approximate-let alone precise-nature is as yet unknown). In this instance, however, several biological mechanisms have been established in animal and cell experiments and now await demonstration as to whether they are valid for humans and have relevance for public health or not. Importantly, if one or more of the suggestive biological mechanisms were to be substantiated for humans, this substantiation could provide a biological rationale for interpreting any associations between shift work and cancers observed in epidemiologic studies as reflecting a cause and effect phenomenon.

\section{A dilemma of shift work and chronodisruption research}

With their 2007 classification, IARC strongly called for epidemiologic research into the biologically probable nexus of shift work involving chronodisruption and two of the three most epidemic cancers in Germany and worldwide, namely, breast and prostate cancer (GLOBOCAN 2002. www-dep.iarc.fr/globocan/database.htm, 8, 9, 10). Empirically, recent meta-analyses evinced that-in line with predictions following from the chronodisruption hypothesis or theory-not only breast cancer [the summary relative risks for investigations of flight personnel and shift workers suggested a respective $70 \%$ and $40 \%$ increase, respectively, in the risk (6)], but also prostate cancer risks appear to be significantly increased [summary relative risks for investigations of flight personnel conveyed a $40 \%$ increase in the risk (6)], likely exposed to chronodisruption via transmeridian, time-zone travel and shiftwork conditions. On the other hand, a large cohort study of cancer risk in Sweden, published only in October of 2007 and thus not included in these metaanalyses, found no evidence for an association between shift work and breast or prostate cancer (11). However, the authors, and an accompanying editorial (12), pointed to shortcomings of this ecological study, in particular with regard to the possible misclassification of exposure as a consequence of the used aggregated, rather than individual, exposure information.

The exposure pitfall actually highlights the problem that IARC insights published so far (detailed results are envisaged for volume 98 of the IARC monograph series) do not provide us with any indication as to what "dose" or precise type of shift work and what "dose" or type and extent of possibly associated chronodisruption may be actually a part of the suspected causal chain(s) leading to cancer developments.

In principle, the observed $70 \%$ increase in the risk of breast cancer among female flight personnel versus a $40 \%$ increase in risk among women who do shift work (6) may be due to the higher "dose of chronodisruption" among the former. In fact, shiftwork conditions that the former may share with the latter could be compounded by rapid "extra-chronodisruption" via extended timezone travel (putting aside a conceivable synergy with or potential confounding by cosmic radiation). In any case, information as to what "dose" of shift work and associated chronodisruption may be relevant could be critical for the identification of prevention strategies.

Indeed there can be no doubt that shift work is inevitable in today's world-at work and at home. But, equally clearly, in a political climate, in which the European Commission decided recently that working up to 65 hours per week can be acceptable (European Commission 2008: available at http://euobserver.com/ 9/26289?rss_rk=1), workers alarmed by the IARC classification are entitled to more insight via epidemiologic studies, be it that these strengthen or, indeed, weaken the overall evidence for a carcinogenicity of shift work involving chronodisruption (13).

Against this background, a dilemma of research on shift work and chronodisruption certainly is that we must strive to arrive at some answers regarding the "probable" causation of human cancers both reliably and quickly. As prospective studies might take decades to provide definitive observational insights, a promising alternative 
may be to use retrospective investigations. Ideally, such studies would not only investigate possible risk relationships between shiftwork conditions, chronodisruption, and cancer in their own right, but should also help, in a time-efficient way, shape further studies. Indeed, so far little information is available, and none has been published by IARC on the type of shift work that would be the likely culprit, apart from shiftwork patterns, in which those "including nightwork are the most disruptive for circadian rhythms [p 1065]" (1). To take but one example, it is not even unambiguously settled whether forward or backward shift rotation is less favorable (14) although there is some "support for the use of forwardrotating shift systems in preference to backward-rotating shift systems [p 197 in 15]" (15-17).

\section{Epidemiologic research options}

In the chemical, steel, and automobile industries, in which the workforce is predominantly male, there are only three studies to date with limited information on prostate cancer risks $(11,18,19)$. Yet, since no occupational confounders are known $(10,20)$, prostate cancer studies appear to offer a good opportunity with which to investigate the effects of chronodisruption.

It is therefore proposed that well-defined and welldocumented occupational study populations be revisited with the objective of employing as much information on work regimes as possible. In this vein, a German research group will try to build upon a coal-miner study $(21,22)$ to explore possible relationships between shift work and the incidence of prostate cancer. Among 4261 coal miners from the Saar area, 87 incident cases of prostate cancer were identified via the Saar cancer registry during a follow-up from 1980 to 2002 (110.4 expected number of cases, standardized incidence ratio of 0.79 , and $95 \%$ confidence interval of $0.63-0.97$ ). The coal miners worked under a rather stable shift system, and documentation about the type of individual shifts appears to be available back to 1980 . The approach is now to go back to this well-monitored Saar cohort and analyze the prostate cancer incidence rates in light of the envisaged shiftwork information. Empirically, what we exemplify with the German cohort has precedence insofar as there was a short contribution by Conlon and his colleagues last year. Instigated by the study of Kubo et al (18) on prostate cancer risks among rotating shift workers, they went back to their case-control study information and offered additional analyses for which they used previously unconsidered information on fulltime rotating shift work (19). It must be emphasized that, when well-defined occupational studies are revisited, the study population information should neither be excluded nor included because the overall relative risk estimate for prostate cancer is below or above 1, respectively. In fact, it is important to obtain unbiased epidemiologic results, irrespective of prior study results without consideration of shiftwork information. If diligent consideration of shiftwork information in the Saar cohort with an overall standardized incidence ratio of 0.79 were to show that a significant proportion of coal miners were engaged in shift work, then this study population might provide evidence against shift work being a risk factor for prostate cancer.

The suggested industry-based cohort studies should be complemented by population-based cohort and casecontrol studies. However, since exposure information will likely be obtained exclusively via a questionnaire rather than from company records, the latter should be viewed as hypothesis-generating and hypothesis-focusing options rather than as investigations to assess precise risks. For example, questionnaire-based case-control studies can be prone to additional self-selection bias among the controls and to recall bias among the cases (23). On the other hand, population-based studies can be helpful to look at a wider range of shift work in different types of jobs and different socioeconomic groups.

Moreover, for effective research into the possibly causal nexus of shift work, chronodisruption, and human cancers, it is desirable to develop biomarkers, both for studying the possible links and for showing the effectiveness of means of preventing chronodisruption. Preferably, these biomarkers must not only allow the assessment of the presence or absence of chronodisruption, but also the quantification or dose of chronodisruption. We should therefore identify biomarkers that enable both the "backward" process of associating it with exposure to shift work and the "forward" process of linking it with cancer and other adverse health effects. Note that there can be numerous biomarkers that may convey facets of a disrupted physiology and could thus be useful as a biomarker of exposure (candidates include body temperature, melatonin, cortisol) but may have a low or no predictive value as a biomarker of the diseases in question, namely, breast and prostate cancer. Alternatively, there could be biomarkers of effect that are expressed long after exposure, and they may prove to be very useful in predicting the onset and progression of disease or for calculating risk. Finally, biomarkers could be markers of susceptibility that describe the ability of a person to cope with exposure. Because the role of candidate biomarkers (exposure, disease, or susceptibility marker) is usually unknown, an appropriate analysis is difficult or even impossible (23). Animal models should be employed to provide an understanding of the mechanistic bases of the expression of markers and the relationships between the continuum (early and late effects) from exposure to disease and to develop hypotheses about how to use the biological markers in epidemiologic studies. 
Elsewhere, it has already been proposed that IARC or the National Institute of Environmental Health Sciences (24) convene a panel of experimental and epidemiologic researchers to develop shiftwork and chronodisruption exposure metrics that are valid for epidemiologic research into chronodisruption and cancer and other diseases $(13,25)$ and could allow a comparison and merging of individual study results. Such authoritative suggestions on possibly relevant details of shift work and chronodisruption would have to be based on theoretical and practical considerations; the candidate information could include length, type, direction, and regularity, but also the workers' ages, medication, tolerance, exercise, and the like. In addition, please note that there is recent, intriguing evidence that, beyond a lightdependent circadian clock, there is a second circadian master clock that is governed by food availability (26). What was long-anticipated by shiftwork researchers earlier, namely, that food provision can play a critical Zeitgeber role in the entrainment of workers' biological 24-hour rhythms to shiftwork conditions, now has a neuroanatomical correlate in another region of the aforementioned hypothalamus, namely, a second circadian master clock appears to be located in the dorsomedial nucleus of the hypothalamus. This finding actually implies that epidemiologists may also have to assess, for instance, via appropriate questionnaire information, when and what shift workers have eaten (retrospective studies) and eat (prospective studies). But there could be even more to consider in terms of when to act and when to study.

\section{A case for preventive action?}

According to the contributors to the Cologne Colloquium 2008, there can be no doubt that today's evidence of both a light- and food-entrainability of circadian rhythms should not only soon lead to targeted research, but also to the development of possibly preventive measures for shift workers. With regard to suspected fooddependent circadian clues, Stokkan and his colleagues (27) suggested, already in 2001, that "Major complaints of shift workers and time zone travelers often involve gastrointestinal symptoms, which might be alleviated by careful attention to meal timing [p 492]" (27). With the group 2A classification of IARC for shift work involving circadian or chronodisruption as a probable human carcinogen, the case for preventive actions seems even stronger today. In fact, we should not have to wait until we have definitive "scientific proof" that the biologically plausible links between chronodisruption, endocrine systems, and cancers in shift workers are causal but could develop means of prudent avoidance of chronodisruption now. A quote from Sir Bradford Hill may be in order here: "All scientific work is incomplete—whether it be observational or experimental. All scientific work is liable to be upset or modified by advancing knowledge. That does not confer upon us a freedom to ignore the knowledge we already have or to postpone the action that it appears to demand at a given time [p 300]" (28).

In the latter vein, examples as to how to alleviate chronodisruption, and its effects, were provided by Foster \& Kreitzman (14) when they suggested coping with jet lag and, by implication, shift lag and possible chronodisruption by seeking out or hiding from daylight or by taking melatonin at a particular time of day. This advice may-and possibly should — soon be complemented by a practical focus on the second critical cue to circadian rhythmicity, namely, when and what to eat as a shift worker.

But, most importantly, in addition to the consideration of relatively novel insights, possible means of mitigating or preventing chronodisruption can build on abundant shiftwork research over many decades. Indeed, the IARC classification should help to overcome the sometimes irrational resistance of those who work at unusual times in shifts and at night with respect to the sound advice as to how to design shiftwork schedules more appropriately for workers of different ages (ie, with presumably less adverse health effects). For example, shift workers should be asked about when they prefer to sleep so that work schedules can be allocated accordingly $(29,30)$.

\section{Conclusions of the Cologne Colloquim Contributors}

Taken together, when IARC concluded in October of 2007 that "shift work that involves circadian disruption is probably carcinogenic to humans [p 1065]", it was taken by the contributors to the Cologne Colloquium as a call for both epidemiologic research and, implicitly, preventive measures. Unfortunately, the full IARC report providing additional background to and more details on their decision beyond what was published in Lancet Oncology (1) will take some time to appear in print. However, the participants at the Cologne Colloquium 2008 (IARC 2007: Shift Work, Chronodisruption and Cancer? Questions for Occupational Medicine) had no doubt that the available evidence suggests that this topic will be an area of extensive interest, debate, and needs for possible mitigation in coming years. Indeed, there are two driving forces for future research, fascinating experimental work to elucidate the interfaces between light, biological rhythms, and downstream beneficial and adverse effects on one hand and the epidemiologic necessity to investigate the disconcerting possibility that breast and prostate cancers, and other disease end points, could be causally associated with widespread shiftwork conditions on the other. Key results of this meeting are summarized in the following 10 theses. 
Thesis 1. Circadian or chronodisruption and possible causal links with diseases, including cancers, constitute a global challenge for the future (31). Experimental evidence of the links has been established in animal models.

Thesis 2. As a basis for both necessary research and the development of means of prevention, we will need to identify which aspects, if any, of shift work, chronodisruption, and individual susceptibility can be relevant to the postulated chain(s) of causation that may lead to human cancer.

Thesis 3. The results of six of eight epidemiologic studies indicating a statistically significant, albeit moderate, increase in the risk of breast cancer among shift workers await confirmation. Moreover, in line with an IARC press release (International Agency for Research on Cancer press release no 180, 5 December 2007. Available at: http://www.iarc.fr/en/Media-Centre/IARC-PressReleases/Communiques-recents/IARC-MonographsProgramme-finds-cancer-hazards-associated-with-shiftwork-painting-and-firefighting), we do need studies that examine potential risks beyond "breast cancer" and in professions beyond "nurses and flight attendants". To this end, research groups should investigate the possibility of adding shiftwork information, as detailed as possible, to their established industry-based databases and using them in further analyses of prostate (or other) cancer risks. At the very least, this could be an option for defining study variables describing chronodisruption due to shift work and to test the appropriateness of such variables. Ultimately, this initiative may help to meet the challenge identified by IARC in October of 2007, namely, to conduct targeted studies and to develop possible means of prevention.

Thesis 4. Population-based studies with retrospective exposure assessment via questionnaires or interviews should complement industry-based epidemiologic research using exposure data that is documented in an independent fashion. They will likely be heuristic or hypothesis-generating and hypothesis-refining in nature rather than yielding precise risk estimates.

Thesis 5. More generally, it must be rigorously investigated whether biological candidate mechanisms that have been identified in animal and cell experiments are at work in humans and have relevance for public health or not. More specifically, for effective research into the possible causal nexus of shift work, chronodisruption, and human cancers, it is desirable to develop biomarkers. They could be a critical means to the following two ends: (i) studying the possible links and (ii) showing the effectiveness of measures for preventing chronodisruption. However, general limitations of the use of markers in epidemiologic studies should be considered.

Thesis 6. Experimental and epidemiologic researchers should consult with shiftwork researchers to identify, in an a priori fashion, possible shiftwork exposures that are more or less prone to lead to chronodisruption. Excessive modeling of shift exposure metrics could lead to data dredging and should be avoided (32). IARC or the National Institute of Environmental Health Sciences could convene a panel of researchers to develop shiftwork and chronodisruption exposure metrics that are valid for observational studies into chronodisruption and cancer, and other diseases. For instance, it is imperative to record more precisely, in a systematic fashion, shiftwork schedules worked and the time actually spent in such regimes.

Thesis 7. Occupational physicians should consult with shiftwork researchers about what shift work regime is the most favorable with regard to reducing or eliminating chronodisruption and as to how to persuade shift workers of the necessity and health benefits of such regimes.

Thesis 8. Female and male workers may benefit from preventive measures via shift organization not only with regard to the putative, albeit as yet not definitive, links between chronodisruption and cancer, but also with regard to the well-documented short- and medium-term adverse health effects of chronodisruption, such as sleep or gastrointestinal disturbances.

Thesis 9. Occupational physicians should be diligently informed about the 2007 IARC classification, its scope, its current limitations, and its future implications.

Thesis 10. The German Society for Occupational Medicine and Environmental Medicine (DGAUM) should consider having a working group that coordinates systematic and concerted responses to the IARC challenge, with regard to both research and preventive measures. In a near(er) future, medical screening does not appear to be a preventive option.

\section{Acknowledgments}

The contents of this manuscript were developed and discussed by the nine Cologne Colloquium presenters at the Universität zu Köln on 16 September 2008 and synthesized by TCE and PM. Comments by Lin Fritschi on an earlier draft are gratefully acknowledged.

Part of this article is based on a presentation made at the 20th International Conference on Epidemiology 
in Occupational Health (EPICOH), 9-11 June 2008, in Costa Rica.

TCE gratefully acknowledges support from the Lieselotte und Dr Karl Otto Winkler-Stiftung für Arbeitsmedizin.

\section{References}

1. Straif K, Baan R, Grosse Y, Secretan B, Ghissassi FEL, Bouvard $\mathrm{V}$, et al. Carcinogenicity of shift-work, painting, and firefighting. Lancet Oncol. 2007;8:1065-6.

2. Gery S, Koeffler HP. The role of circadian regulation in cancer. Cold Spring Harb Symp Quant Biol. 2007;72:459-64.

3. Reppert SM, Weaver DR. Coordination of circadian timing in mammals. Nature. 2002;418(6901):935-41.

4. Weaver DR: The suprachiasmatic nucleus: a 25-year retrospective. J Biol Rhythms. 1998;13(2):100-12.

5. Fu L, Lee CC. The circadian clock: pacemaker and tumour suppressor. Nat Rev Cancer. 2003;3:350-61.

6. Erren TC, Pape HG, Reiter RJ, Piekarski C. Chronodisruption and cancer. Naturwissenschaften. 2008;95:367-82.

7. Greenland S, Gago-Dominguez M, Castelao JE. The value of risk-factor ("black-box") epidemiology. Epidemiology. 2004;15:529-35.

8. Parkin DM, Whelan SL, Ferlay J, Teppo L, Thomas DB, editors. Cancer incidence in five continents, vol VIII. Lyon (Paris): International Agency for Research on Cancer; 2002. IARC scientific publication, no 155.

9. Robert Koch-Institut und die Gesellschaft der epidemiologischen Krebsregister in Deutschland eV, editors. Krebs in Deutschland 2003-2004: Häufigkeiten und Trends. 6. Überarbeitete Auflage [Cancer in Germany 2003-2004: Frequencies and trends. 6th revision]. Berlin: Robert Koch-Institut und die Gesellschaft der epidemiologischen Krebsregister in Deutschland $\mathrm{eV} ; 2008$.

10. Mucci AL, Signorello LB, Adami HO. Prostate cancer. In: Adami HO, Hunter D, Trichopoulos D, editors. Textbook of cancer epidemiology. 2nd ed. Oxford, New York: University Press; 2008. p 517-54.

11. Schwartzbaum J, Ahlbom A, Feychting M. Cohort study of cancer risk among male and female shift workers. Scand J Work Environ Health. 2007;33(5):336-43.

12. Pukkala E, Härmä M. Does shift work cause cancer? [editorial]. Scand J Work Environ Health. 2007;33(5):321-3.

13. Erren TC, Reiter RJ, Piekarski C. Chronodisruption and melatonin: the need for sensible exposure metrics in epidemiological studies. J Pineal Res. 2008;45(3):335-6.

14. Foster R, Kreitzman L. The biological clocks that control the daily lives of every living thing: rhythms of life. London: Yale University Press profile books; 2004.

15. Driscoll TR, Grunstein RR, Rogers NL. A systematic review of the neurobehavioural and physiological effects of shiftwork systems. Sleep Med Rev. 2007;11:179-94.

16. Knauth P. Speed and direction of shift rotation. J Sleep Res. 1995;4(S2):41-6.

17. Viitasalo K, Kuosma E, Laitinen J, Härmä M. Effects of a rapidly forward and a flexible backward rotating shift-systems on employee's daytime alertness and cardiovascular risk factors. Shiftwork News. 2007;24(2);107.

18. Kubo T, Ozasa K, Mikami K, Wakai K, Fujino Y, Watanabe $\mathrm{Y}$, et al. Prospective cohort study of the risk of prostate cancer among rotating-shift workers: findings from the Japan collaborative cohort study. Am J Epidemiol. 2006;164(6):549-55.

19. Conlon M, Lightfoot N, Kreiger N. Rotating shift work and risk of prostate cancer. Epidemiology. 2007;18:182-3.

20. Fritschi L, Glass DC, Tabrizi JS, Leavy JE, Ambrosinsi GL. Occupational risk factors for prostate cancer and benign prostatic hyperplasia: a case-control study in Western Australia. Occup Environ Med. 2007;64:60-5.

21. Morfeld P, Lampert K, Ziegler H, Stegmaier C, Dohm G, Piekarski C. Coal mine dust exposure and cancer mortality in German coal miners. Appl Occup Environ Hyg. 1997;12:909-13.

22. Morfeld P, Stegmaier C, Emmerich M, Piekarski C. Krebsmorbidität und Krebsmortalität saarländischer Steinkohlenbergleute in Abhängigkeit von Staubexposition und Pneumokonioseentwicklung (Phase IV): Abschlussbericht zum Forschungsvorhaben der Arbeitsgemeinschaft des Saarlandes zur Erforschung und Förderung des Gesundheitsschutzes im Bergbau e.V. [Cancer morbidity and mortality of coalminers from the Saar area in dependence of dust exposures and the development of pneumoconiosis (phase IV): final report on the research project of the AGIB]. Saarbrücken (Germany): Alma Mater; 2005. http://cms.uk-koeln.de/live/arbeitsmedizin/content/e159/e256/AbschlussberichtKrebs.pdf.

23. Rothman KJ, Greenland S, Lash TL. Modern epidemiology 3rd ed. Philadelphia (PA): Lippincott Raven; 2008

24. Stevens RG, Blask DE, Brainard GC, Hansen J, Lockley SW, Provencio I, et al. Meeting report: the role of environmental lighting and circadian disruption in cancer and other diseases. Environ Health Perspect. 2007;115:1357-62.

25. Erren TC, Pape HG, Piekarski C, Reiter RJ. Not all shifts are equal: it's time for comprehensive exposure metrics in chronodisruption research. Cancer Res. 2008;68:4011.

26. Fuller PM, Lu J, Saper CB. Differential rescue of light- and food-entrainable circadian rhythms. Science. 2008;320:10747.

27. Stokkan KA, Yamazaki S, Tei H, Sakaki Y, Menaker M. Entrainment of the circadian clock in the liver by feeding. Science. 2001;291:490-3.

28. Hill AB. The environment and disease: association or causation? Proc R Soc Med. 1965;58:295-300.

29. Roenneberg T, Wirz-Justice A, Merrow M. Life between clocks: daily temporal patterns of human chronotypes. J Biol Rhythms. 2003;18:80-90.

30. Gauderer PC, Knauth P. Pilot study with individualized duty rotas in public transport. Trav Hum. 2004;67:87-100.

31. Erren TC. Identifying research challenges for occupational and environmental medicine until 2030: an initiative. Occup Environ Med. 2009;66(1):5-6.

32. Burnham KP, Anderson DR. Model selection and multimodel inference: a practical information-theoretic approach. 2nd ed. New York (NY): Springer Science + Business Media, LLC; 2002.

Received for publication: 7 October 2008 